\title{
Motivation vom Heidi
}

\author{
Jürg Kesselring \\ Prof. Dr. med., Mitglied FMH, Chefarzt Neurologie, Rehabilitationszentrum Valens
}

Natürlich ist es für Leute, die gerne in Nature und Science publizieren, nicht grad üblich, sich im Kinderbuch Heidi von Johanna Spyri zu orientieren, um etwa Hinweise und Anleitungen zur Motivation zu suchen. In der Rehabilitation betrachten wir es ja als ein Hauptanliegen, dass wir die Patientinnen und Patienten motivieren können, die vielfältigen Therapien durchzuführen mit dem Ziel, ihre Selbständigkeit und ihre Lebensqualität zu verbessern. Motivation ist aber auch ein zentral wichtiges Thema für jede und jeden von uns im Alltag. Gut dargestellt finde ich es im allseits bekannten Buch Heidi und besonders schön illustriert im Spielfilm (Dezember 2015) von Alain Gsponer mit Bruno Ganz und Anuk Steffen: Der Geissenpeter, der tüchtig seine Geissen hütet und fröhlich pfeifen kann, aber keinen ganzen Satz zustande bringt, sagt bezüglich des Lesens und Schreibens zum Heidi, das sei viel zu schwer und nütze sowieso nichts. Im noblen Hause Sesemann in Frankfurt soll Heidi unterrichtet werden und dazu Lesen und Schreiben lernen. Die hochnäsige, klugscheissende Erzieherin, Fräulein Rottenmeier, die aber ein Angsthase ist, wie es für diese Art von Besserwissern typisch ist, ist entsetzt, dass «dieses Wesen nicht einmal die Urbegriffe kennt» (z.B. «Frühstück» ...). Der Lehrer, der hier immer nur «der Herr Kandidat» genannt wird, versucht geduldig, aber auch ziemlich irritiert, das Alphabet beizubringen, wobei Heidi in den schwarzen Bögen und Linien und Punkten der Buchstaben lediglich Formen zu erkennen vermag wie Geissbockhörner, Falknis-Motive, Sonnenuntergänge etc. Klaras gütige Grossmama in Frankfurt aber zeigt ihm im Bilderbuch, dass es von den Bildern mehr verstehen könne, wenn es selber die Legenden zu lesen vermöchte, und dann nicht immer andere fragen müsste, sondern den Text selbst befragen könne und damit etwas für sich selber lerne. Jedes Kind in Russland lernt das kurze Gedicht «Kak charasho umet citat ...»: "Ach wie schön ist es, selber lesen zu können: man braucht dann nicht mehr die Oma und auch nicht das Schwesterlein zu bitten, vorzulesen. Man nimmt einfach selber ein Buch aus dem
Gestell und liest für sich oder für andere ...» Und vor allem sagt die Grossmama in Frankfurt, wäre Heidi dann auch imstande, der geliebten blinden Grossmutter von Peter zu Hause vorzulesen und ihr damit das Gefängnis ihrer Behinderung durchbrechen und öffnen zu können. Das ist dann Heidis Motivation, und es lernt im Nu zu lesen und nimmt in Kauf, dass einer wie der Geissenpeter das nur wichtigtuerisch findet und wie so viele von dieser Art mit faulen Ausreden («keine Zeit», «anderes zu tun», «sowieso nur für Streber» etc.) und sturen Behauptungen (z.B. «es gibt viel mehr Leute, die auch nicht lesen und schreiben können, warum soll dann gerade ich das machen?», «bin halt so schüchtern») hantiert und sich nach der wirklichen Motivation nicht einmal erkundigt oder gar böswillig falsche unterstellt. Jede/r kann selber wählen, wo und bei wem er/sie aufbauende Motivation und Ermunterung erfährt und bei wem eher geissenpeterische $\mathrm{Zu}$ friedenheit mit dem, was halt grad da ist, und mit der Bemerkung, dass «die anderen auch nur mit Wasser kochen». Es kommt eben darauf an, was man mit seinem Wasser macht.

\section{Heidi lernt im $\mathrm{Nu}$ zu lesen und nimmt in} Kauf, dass einer wie der Geissenpeter das nur wichtigtuerisch findet.

Paul Good, Philosoph in Bad Ragaz, hat seine «Philosophie vom kleinen Weltstar Heidi» geschrieben [1], in der er feinfühlig den sprachlichen Besonderheiten im Text nachspürt, in die Töne und Klänge hineinhört, die zur Lebensmelodie werden. Er nennt es ein «literarisches Meisterwerk» und begründet dies überzeugend. Es steckt eben mehr dahinter, als was die Vermarktungsindustrie aus dem Heidi heute macht, und Paul Good empfiehlt, «sich mit der Originalfigur hinter dem Mythos stärker auseinanderzusetzen». Es lohnt sich!

\footnotetext{
Literatur

1 Paul Good: Heidi. Meine Philosophie vom kleinen Weltstar. Agon Press 2016
} 